\title{
Details of an imaging atom probe
}

\author{
V R SESHADRI, $K$ NEELAKANTAN, E MOHANDAS and \\ V S RAGHUNATHAN
}

Reactor Research Centre, Kalpakkam 603 102, India

\begin{abstract}
An imaging atom probe has been incorporated in a conventional field ion microscope in our laboratory. The principle and instrumentation of the probe has been discussed.
\end{abstract}

Keywords. Field ion microscope; imaging atom probe; high voltage pulser.

\section{Introduction}

An alternative to the field ion microscope atom probe (FIMAP) is the imaging atom probe (IAP) (Panitz 1974). An IAP allows the estimation of chemical composition over a large area of a field ion specimen, while maintaining the excellent spatial resolution of the original atom probe developed by Muller et al (1968). This makes the IAP very much suitable in cases where the composition changes rapidly over a distance of few atom diameters - a situation often encountered at interphase interfaces, grain boundaries with segregation and other lattice inhomogenities. Section 2 gives the principle of an IAP. Section 3 describes the instrumentation and section 4 presents the experimental observations.

\section{Principle}

In a conventional FIM the atoms of the specimen surface can be field evaporated as ions by applying high voltage pulses of few nsec width over the standing d.c. potential. The disorbed species drift towards the detector at a velocity determined by their mass to charge ratio and the total applied potential to the specimen for field evaporation. The detector is then gated on after a delay which corresponds to the drift time of a particular species. The image on the screen then gives the distribution of the selected species over the specimen surface.

\section{Instrumentation}

Figure 1 shows the schematic of an IAP. The microscope is a conventional FIM comprising of a stainless steel vacuum chamber, vacuum pumping system, cryostat for cooling the specimen and a CEMA for image intensification.

The heart of the special instrumentation for IAP is the high voltage pulsers, shown schematically in figure 2. Unlike the conventional pulsers using krytrons or mercurywetted relays these pulsers use a novel method for generating pulses of $\approx 1.1 \mathrm{kV}$ 


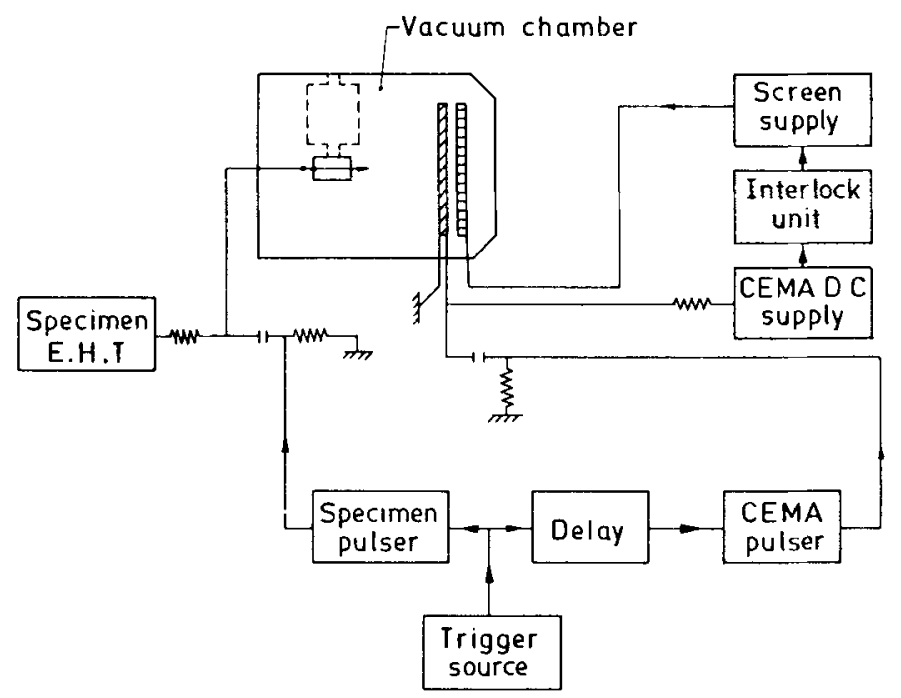

Figure 1. Block diagram of IAP.

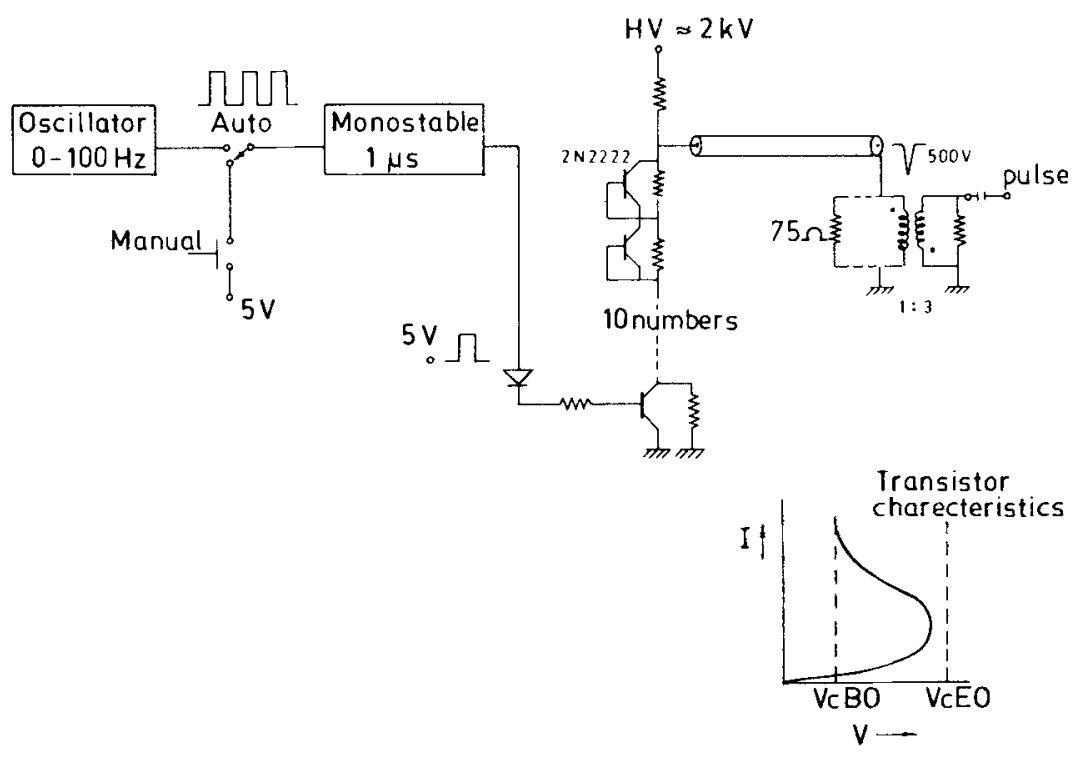

Figure 2. Schematic of high voltage pulser.

amplitude with pulse widths of a few nsec. The circuit is completely solid state and uses the controlled avalanche breakdown of a chain of $n-p-n$ transistors. Ten transistors (2N2222) are connected in cascade as shown in figure 2 . The circuit is biased by a $1.5-2 \mathrm{kV}$ power supply through a $120 \mathrm{k} \Omega$ resistor in such a manner that the $V_{C E}$ of the individual transistors are very close to the collector-base break down voltage. When a TTL ( $5 \mathrm{~V}$ ) pulse is applied to the base of the transistor, at the bottom of the chain it saturates and the applied voltage gets redistributed among the remaining transistors. 
This redistributed voltage is arranged to be greater than the breakdown voltage so that the entire chain goes into the avalenche breakdown mode. The sustaining $V_{C E}$ of the transistors under avalanche breakdown is less than the breakdown voltage itself, because the transistor characteristics fold over (Millman and Taub 1965). Thus a negative pulse appears at the collector of the transistor at the top of the chain. This pulse is too wide to be used directly and is about $1 \mathrm{kV}$ in amplitude. The rise time of the pulse is also quite large though fall time is to the order of a few nsec. This pulse is then shaped by a delay line differentiator and then stepped up by a $1: 2$ pulse transformer to obtain a positive pulse of up to $1.1 \mathrm{kV}$ amplitude with a rise time and fall time of $\approx 5$ nsec.

Two such pulse generators are used and sequentially triggered. The trigger pulses to the pulse generators are delayed by means of a delay generator which uses a monoshot to generate delays upto $1 \mathrm{nsec}$ in steps of $10 \mathrm{nsec}$. The two generators are isolated and completely shielded from each other to prevent spurious triggering of second pulser. The CEMA pulser has to drive a capacitance load of approximately $400 \mathrm{pF}$ and hence appropriate compensation networks are also incorporated in the design. The high voltage pulses with delay are shown in figure 3.

\section{Experimental observation}

The pulses have been connected to the system to achieve the following results:

(i) By applying the pulse to the specimen, superimposed on the operating d.c. voltage, controlled field evaporation has been observed. This helps primarily in shaping the specimen to get the field evaporated end form without specimen failure as is often the case with uncontrollable d.c. field evaporation.

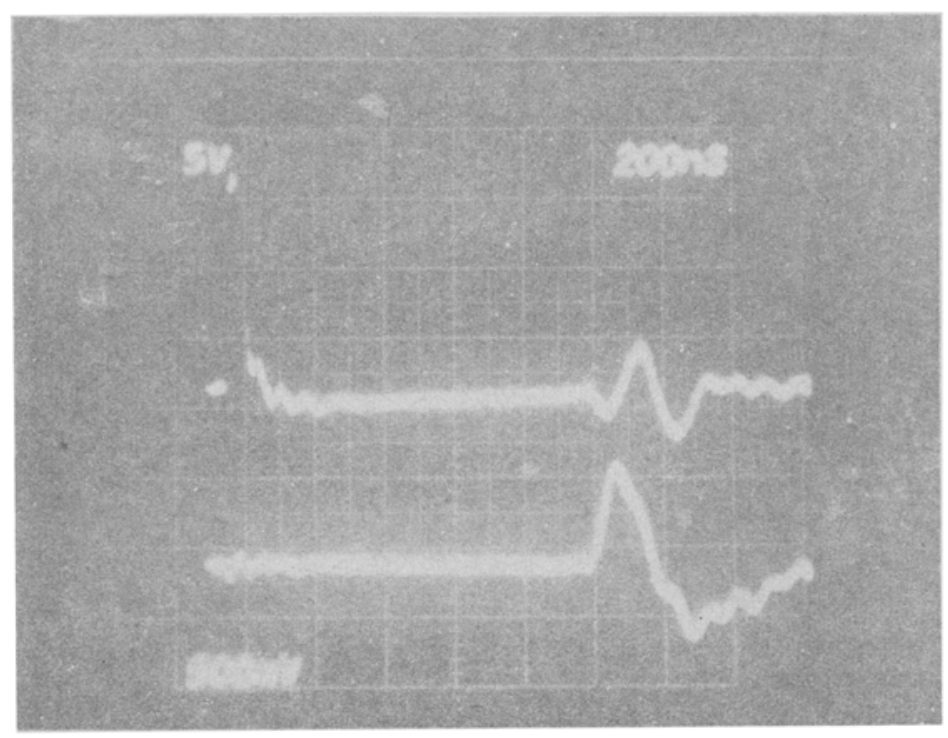

Figure 3. High voltage pulses showing the main pulse (top one at the beginning) and the delayed one (bottom). The main pulse is $\approx 1.5 \mathrm{kV}$ and delayed pulse $\approx 500 \mathrm{~V}$. The delay is 200 nsec div. 
(ii) With pulse applied to CEMA, gated FIM image has been observed with extremely low gas pressure. Further experiments to get IAP pictures were not successful due to poor detector performance.

\section{Conclusion}

A new method of producing high voltage pulser has been described. Application of these pulsers for construction of an IAP is discussed.

\section{References}

Millman J and Taub H 1965 Pulse, digital and switching wave forms (Kagakusha: McGraw Hill)

Muller E W, Panitz J A and Mclane S B 1968 Rev. Sci. Instrum. 3983

Panitz J A 1974 J. Vac. Sci. Technol. 11206 